\title{
Molecular surveillance of Pfcrt and $k 13$ propeller polymorphisms of imported Plasmodium falciparum cases to Zhejiang Province, China between 2016 and 2018
}

\author{
Xiaoxiao Wang ${ }^{1,2 \dagger}$, Wei Ruan ${ }^{2 \dagger}$, Shuisen Zhou ${ }^{1 *}$, Fang Huang ${ }^{1 *}$, Qiaoyi Lu $^{2}$, Xinyu Feng ${ }^{1}$ and He Yan ${ }^{1}$
}

\begin{abstract}
Background: Resistance to anti-malarial drugs hinders malaria elimination. Monitoring the molecular markers of drug resistance helps improve malaria treatment policies. This study aimed to assess the distribution of molecular markers of imported Plasmodium falciparum infections.

Methods: In total, 485 P. falciparum cases imported from Africa, Southeast Asia, and Oceania into Zhejiang province, China, from 2016 to 2018 were investigated. Most were imported from Africa, and only a few cases originated in Asia and Oceania. Blood samples were collected from each patient. Plasmodium falciparum chloroquine resistance transporter (PfCrt) at residues 72-76 and Kelch13-propeller (k13) were determined by nested PCR and DNA sequence.

Results: Wild-type Pfcrt at residues 72-76 was predominant (72.61\%), but mutant and mixed alleles were also detected, of which CVIET (22.72\%) was the most common. Mutant Pfcrt haplotypes were more frequent in patients from West Africa (26.92\%), North Africa (25\%), and Central Africa (21.93\%). The number of cases of P. falciparum infections was small in Southeast Asia and Oceania, and these cases involved Pfcrt mutant type. For the $k 13$ propeller gene, 26 samples presented 19 different point mutations, including eight nonsynonymous mutations (P441S, D464E, K503E, R561H, A578S, R622I, V650F, N694K). In addition, R561H, one of the validated SNPs in k13, was detected in one patient from Myanmar and one patient from Rwanda. A578S, although common in Africa, was found in only one patient from Cameroon. R622I was detected in one sample from Mozambique and one sample from Somalia. The genetic diversity of $k 13$ was low in most regions of Africa and purifying selection was suggested by Tajima's D test.

Conclusions: The frequency and spatial distributions of Pfcrt and $k 13$ mutations associated with drug resistance were determined. Wild-type Pfcrt was dominant in Africa. Among k13 mutations correlated with delayed parasite clearance, only the R561H mutation was found in one case from Rwanda in Africa. Both Pfcrt and k13 mutations were detected in patients from Southeast Asia and Oceania. These findings provide insights into the molecular epidemiological profile of drug resistance markers in the study region.
\end{abstract}

Keywords: Drug resistance, $k 13$ propeller, Malaria, Pfcrt

*Correspondence: zhouss@nipd.chinacdc.cn; huangfang@nipd.chinacdc.cn

${ }^{+}$Xiaoxiao Wang and Wei Ruan contributed equally to this work

${ }^{1}$ National Institute of Parasitic Diseases, Chinese Center for Disease Control and Prevention, Key Laboratory of Parasite and Vector Biology, Schistosomiasis and Filariasis, $\mathrm{MOH}$, and WHO Collaborating Centre for Malaria, Shanghai, People's Republic of China

Full list of author information is available at the end of the article

\section{Background}

Malaria is a significant public health problem because of its worldwide distribution and high mortality. It was estimated that there were 219 million cases of malaria globally in 2017, mostly in 15 sub-Saharan African countries and India, representing approximately $80 \%$ of

c) The Author(s) 2020. This article is licensed under a Creative Commons Attribution 4.0 International License, which permits use, sharing, adaptation, distribution and reproduction in any medium or format, as long as you give appropriate credit to the original author(s) and the source, provide a link to the Creative Commons licence, and indicate if changes were made. The images or other third party material in this article are included in the article's Creative Commons licence, unless indicated otherwise in a credit line to the material. If material is not included in the article's Creative Commons licence and your intended use is not permitted by statutory regulation or exceeds the permitted use, you will need to obtain permission directly from the copyright holder. To view a copy of this licence, visit http://creativeco mmons.org/licenses/by/4.0/. The Creative Commons Public Domain Dedication waiver (http://creativecommons.org/publicdomain/ zero/1.0/) applies to the data made available in this article, unless otherwise stated in a credit line to the data. 
the global malaria burden [1]. Of the five malaria species, Plasmodium falciparum caused the most malaria incidence worldwide, accounting for $99.7 \%$ of estimated malaria cases in the World Health Organization (WHO) African region and $62.8 \%$ in Southeast Asia in 2017, and is the causative agent of the most severe forms of the disease [1].

Effective treatment is critical. Since the 1940s, multiple anti-malarial drugs have been developed and used to treat malaria parasites, including chloroquine (CQ), mefloquine, quinine, pyrimethamine, and sulfadoxine. However, the widespread use of these drugs promotes drug resistance, especially chloroquine resistance (CQR). Resistance to CQ occurred in Southeast Asia, South America, and the Western Pacific region in the late 1950s and rapidly spread to malaria-endemic areas, including Africa [2,3]. Mutations in the chloroquine resistance transporter (Pfcrt) located on the P. falciparum digestive vacuole membrane were responsible for CQ treatment failure $[4,5]$. Amino acid polymorphisms at PfCRT amino acid residues $72-76$ were observed in CQR field isolates, whereas CVMNK haplotypes at PfCRT residues $72-76$ were regarded as chloroquine sensitive (CQS) [6, 7]. Other studies revealed that Pfcrt K76 T variants could affect parasite fitness, increase the rate of gametocyte production, and alter the susceptibility to artemisinin-based combination therapy (ACT) $[3,8-10]$. These results highlight the need to monitor the molecular evolution of $P f c r t$.

In view of $P$. falciparum multidrug resistance, the WHO recommended ACT as the first-line treatment for uncomplicated $P$. falciparum malaria in 2006 [11]. However, the detection of artemisinin-resistant P. falciparum in western Cambodia and the border between Cambodia and Thailand in 2008 was a drawback to malaria elimination [12, 13]. Over 200 nonsynonymous P. falciparum Kelch13 ( $k 13$ ) mutations have been reported to date, of which nine variants (F446I, N458Y, M476I, Y493H, R539T, I543T, P553L, R561H, and $\mathrm{C} 580 \mathrm{Y}$ ) were correlated with slow parasite clearance and reduced in vitro drug sensitivity, and over 20 $k 13$ mutations are considered candidates or associated markers [14]. k13 mutations were detected predominantly in the Greater Mekong subregion(GMS) [15]. k13 mutations are rare in Africa, and their profile is highly heterogeneous [16]. The prevalence of nonsynonymous $k 13$ mutations is low in approximately $50 \%$ of African countries [15]. Nevertheless, the increase in drug resistance in Africa could hamper malaria control considering its high morbidity and mortality. Therefore, monitoring mutations associated with artemisinin resistance via delayed parasite clearance globally, but especially in Africa, is critical.
Zhejiang province, located in eastern China, was considered malaria-free in 2018. No indigenous malaria infections have been reported in Zhejiang province since 2011. However, approximately 200 cases are imported every year, especially $P$. falciparum malaria from Africa. In this study, samples were collected from $P$. falciparum cases imported into Zhejiang Province, China, between 2016 and 2018, and molecular surveillance of Pfcrt and $k 13$ was performed to determine the emergence and spread of drug resistance in the countries of origin.

\section{Methods}

\section{Sample collection and DNA extraction}

Zhejiang province located in the Yangtze River Delta region and has many migrant workers travelling from Africa and Southeast Asia each year. Since 2012, all reported cases are imported. Microscope examination and PCR targeting the DNA of the $P$. falciparum multicopy $18 \mathrm{~S}$ ribosomal RNA gene were employed to confirm suspected infections.

In this study, 485 cases of $P$. falciparum imported into Zhejiang Province, China between January 2016 and December 2018 were investigated. Venous blood was obtained from each patient. In total, 485 whole blood samples were collected. All blood samples were stored at $-80{ }^{\circ} \mathrm{C}$ until use. Parasite genomic DNA was extracted from $200 \mu \mathrm{L}$ of blood using the QIAamp DNA Mini kit (QIAGEN Inc., Germany) following the manufacturer's instructions.

\section{DNA amplification and sequencing}

The $k 13$ and $P f c r t$ genes were amplified by nested PCR, as previously described $[17,18]$. The primers for PCR were described in previous study and they are listed in Additional file 1: Table S1 $[17,18]$. Proof-reading polymerase was used in each reaction to prevent amplification errors. The proof-reading polymerase was contained in the High Fidelity PCR Master Mix (item number B639292), which was supplied by Sangon Biotech Co., Ltd. (Shanghai, China). The amplification conditions in both PCR rounds were as follows: one cycle at $95{ }^{\circ} \mathrm{C}$ for $2 \mathrm{~min}$, followed by 30 cycles at $95^{\circ} \mathrm{C}$ for $2 \mathrm{~s}, 60^{\circ} \mathrm{C}$ for $90 \mathrm{~s}$, and $72{ }^{\circ} \mathrm{C}$ for $90 \mathrm{~s}$, and one extension cycle at $72{ }^{\circ} \mathrm{C}$ for $10 \mathrm{~min}$. PCR products were sequenced by Sangon Biotech Co., Ltd. (Shanghai, China).

\section{Data analysis}

Mega version 7.0.26 (https://www.megasoftware.net/) was used to align amplicon sequences to 3D7 reference strain sequences retrieved from the NCBI database. A database was constructed using Microsoft Excel 2017, and statistical analysis was performed with SPSS Statistics for Windows version 21.0 (IBM Corp., Armonk, 
NY, USA). P-distance, the proportion of nucleotide sites at which two sequences being compared are different, was obtained by dividing the number of nucleotide differences by the total number of nucleotides compared. It was estimated by Mega version 7.0.26. Nucleotide diversity (Jukes and Cantor) (Pi (JC)), the average number of nucleotide substitutions per site between two sequences, was obtained using the Jukes and Cantor correction. Tajima's D test was used as a neutrality test. Number of segregating sites (S), haplotype diversity (Hd), Pi (JC), average number of nucleotide differences $(\mathrm{k})$ and Tajima's $\mathrm{D}$ test were performed using DnaSP 6.12.03. Pearson Chi-square test was used for statistical analysis. Variables with a $P$ value smaller than 0.05 were considered statistically significant.

\section{Results}

\section{General information}

A total of 485 P. falciparum cases imported from 37 countries from 2016 to 2018 were included in this study. Most cases involved migrant workers returning from Africa. Blood samples were collected from each patient, and 192, 169, and 124 samples were collected in 2016, 2017, and 2018, respectively (Additional file 1: Table S2). The median (range) age of the study population was 42 (9-69) years. A total of 426 out of 485 patients were men. Most cases were imported from West Africa (54.43\%, 264/485), Central Africa (24.54\%,119/485), and South Africa (12.16\%, 59/485) (Fig. 1). Only a few cases originated in East Africa, North Africa, Philippines, Myanmar and Papua New Guinea, accounting for $6.80 \%(33 / 485)$, $0.82 \%$ (4/485), $0.21 \%$ (1/485), $0.21 \%(1 / 485)$ and $0.82 \%$ (4/485), respectively.

\section{Pfcrt mutations}

The amplified $145 \mathrm{bp}$ fragment of Pfcrt encoded amino acid residues 72 to 76 . Of the 485 samples, 471 were sequenced successfully, whereas 14 isolates [DR Congo $(\mathrm{n}=2)$, Gabon $(\mathrm{n}=2)$, Congo $(\mathrm{n}=1)$, Mozambique $(\mathrm{n}=1)$, Angola $(\mathrm{n}=2)$, Malawi $(\mathrm{n}=1)$, Republic of South Africa $(n=1)$, Guinea $(n=1)$, Nigeria $(n=3)]$ were not sequenced successfully because of the poor quality of DNA (Table 1). Wild-type Pfcrt alleles (CVMNK), mutant Pfcrt alleles (CVIET, SVMNT) and mixed Pfcrt alleles $(\mathrm{CVM} / \mathrm{I} \mathrm{N} / \mathrm{E} / \mathrm{D} / \mathrm{K} \mathrm{K} / \mathrm{T})$ were detected, and the most prevalent alleles were wild-type CVMNK (72.61\%, $342 / 471)$ and mutant $(22.72 \%, 107 / 471)$. Of the mixed haplotypes, the nucleotide sequences corresponding to residues $74-76$ were ATG/T, A/GAA/T, and AA/CA, and could encode different amino acid sequences (CVM/I N/E/D/K K/T) (Table 1, Fig. 2).

\section{Spatial distribution of Pfcrt mutations}

Pfcrt mutations were more common in patients from West Africa (26.92\%, 70/260), North Africa (25.00\%, $1 / 4$ ) and Central Africa (21.93\%, 25/114). The smallest percentage of Pfcrt mutations was found in East Africa (6.06\%, 2/33). The difference in the prevalence of Pfcrt mutations between West, Central, North, East, and South Africa was statistically significant $\left(\chi^{2}=14.165, P<0.05\right)$. The spatial distribution of these mutations was highly heterogeneous. The percentage of wild-type, mutant, and mixed haplotypes varied greatly between countries. Although the number of cases originating in Asia and Oceania was small, these cases had a high rate of the mutant type. One out of two patients returning from Asia presented mutant alleles CVIET and three out of four patients returning from Papua New Guinea harbored SVMNT haplotypes (Table 1, Fig. 1).

\section{k13 propeller mutations}

The nested PCR yielded an 849-bp amplification product. Among the 485 samples, 437 were successfully amplified and sequenced (Central Africa:106, East Africa: 21, North Africa: 4, South Africa: 53, West Africa: 247, Southeast Asia: 2, Oceania: 4). Twenty-six samples presented 19 different point mutations, including eight nonsynonymous mutations (P441S, D464E, K503E, R561H, A578S, R622I, V650F, N694K) (Table 2). DNA sequences with the 19 point mutations were submitted to the NCBI database under GenBank accession numbers MN586239 to MN586257. It is worth noting that an $\mathrm{R} 561 \mathrm{H}$ mutation, one of the nine validated molecular markers associated with for artemisinin resistance via delayed parasite clearance, was detected in two patients, one each from Myanmar and Rwanda. Moreover, two samples harboring a P441S mutation contained either mutant or mixed $P f c r t$ haplotypes (Table 2). The A578S substitution, although common in Africa, was only found in one patient returning from Cameroon, and K503E was identified in another sample. In addition, R622I was detected in two samples, one each from Mozambique and Somalia. V650F, N694K, and D464E substitutions were present in parasite strains from Nigeria, Côte d'Ivoire, Angola, and Guinea, respectively.

\section{Genetic diversity of $k 13$}

The genetic diversity of the $k 13$ gene represented by the $\mathrm{P}$-distance, $\mathrm{S}, \mathrm{Hd}, \mathrm{Pi}$, and $\mathrm{k}$ values was low in the imported samples (Table 3). Similarly, only 15 haplotypes were observed in isolates imported from West Africa, with a haplotype diversity of 0.141 . The patients from East Africa only had two haplotypes, but the 


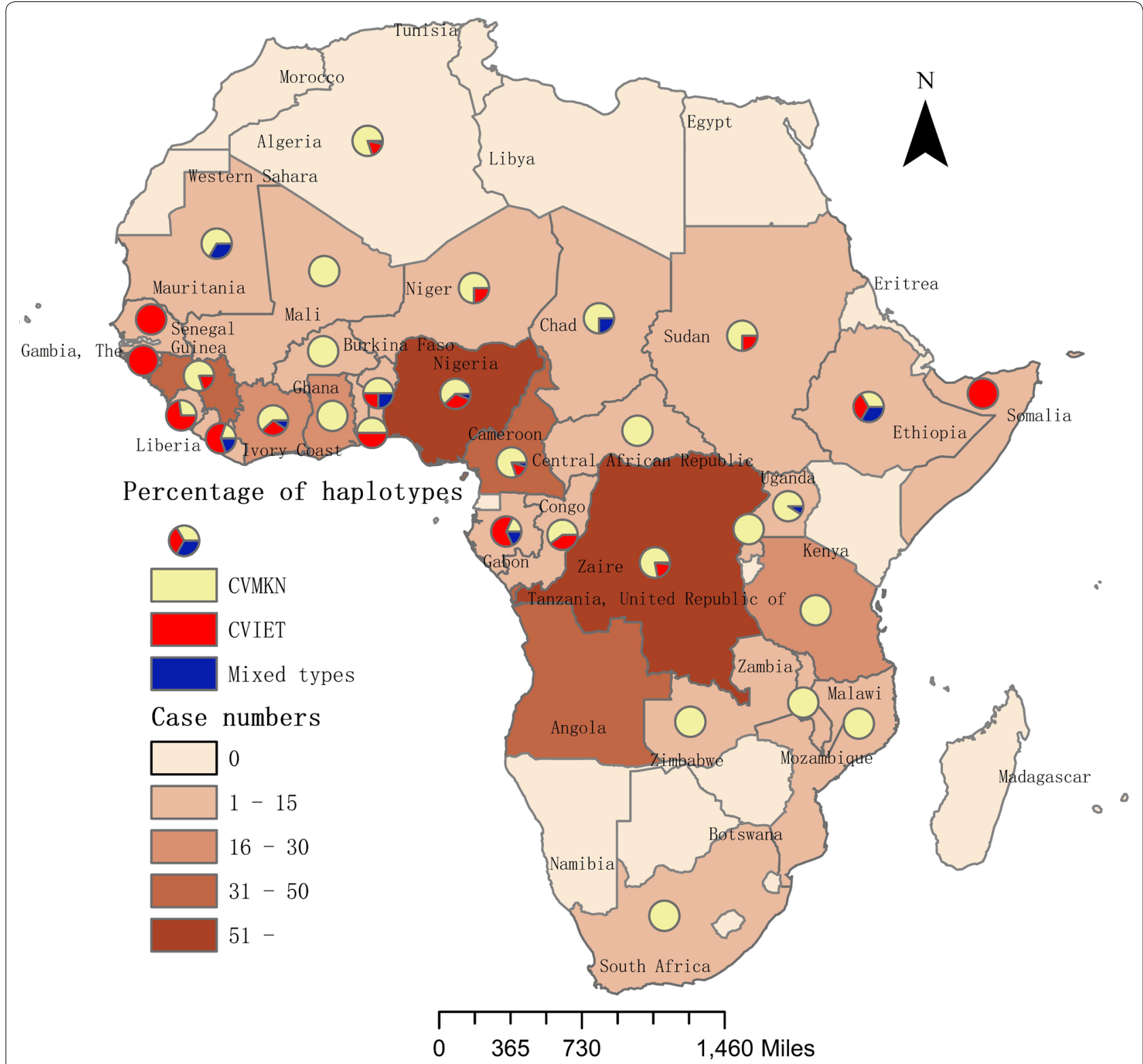

Fig. 1 The number of imported P. falciparum cases from Africa and percentage of haplotypes of Pfcrt

haplotype diversity, nucleotide diversity, and average number of nucleotides were the highest compared with those from other areas. After combining all the samples, Tajima's D significantly deviated from neutrality. However, when the samples from the different regions were analysed separately, the negative Tajima's D on $k 13$ was only evident in the isolates from West Africa. The Tajima's D values for the samples from South Africa, Central Africa, and East Africa were not statistically significant, but they exhibited a similar trend to that obtained for the samples from West Africa.

\section{Discussion}

As one of the most important approaches to combat malaria, chemotherapy is paramount to treat Plasmodium and interrupt malaria transmission. However, effective anti-malarial drug policies were followed by drug resistance, which temporarily interrupted malaria elimination campaigns. Drug resistance has posed a significant threat to global malaria control strategies and raised international concern, especially in areas most strongly affected by the disease [19]. Most malaria cases originated in the WHO African region, accounting for approximately $92 \%$ of all cases and $93 \%$ deaths [1]. Four 
Table 1 Prevalence of Pfcrt genotypes between 2016 and 2018

\begin{tabular}{|c|c|c|c|c|c|}
\hline \multirow[t]{2}{*}{ Region } & \multirow[t]{2}{*}{ Country } & \multirow[t]{2}{*}{ No } & \multicolumn{3}{|l|}{ Prevalence (\%) } \\
\hline & & & Wild type (CVMNK) & $\begin{array}{l}\text { Mutation type } \\
\text { (CVIET/SVMNT) }\end{array}$ & $\begin{array}{l}\text { Mixed type } \\
\text { (CVM/I } \\
\text { N/E/D/K K/I) }\end{array}$ \\
\hline \multirow[t]{7}{*}{ Central Africa } & & 114 & $83(72.81)$ & $25(21.93)$ & $6(5.26)$ \\
\hline & DR Congo & 50 & $39(78.00)$ & $10(20.00)$ & $1(2.00)$ \\
\hline & Cameroon & 40 & $32(80.00)$ & $6(15.00)$ & $2(5.00)$ \\
\hline & Central African Republic & 4 & $4(100.00)$ & $0(0)$ & $0(0)$ \\
\hline & Chad & 4 & $3(75.00)$ & $0(0)$ & $1(25.00)$ \\
\hline & Congo & 5 & $3(60.00)$ & $2(40.00)$ & $0(0)$ \\
\hline & Gabon & 11 & $2(18.18)$ & $7(63.64)$ & $2(18.18)$ \\
\hline \multirow[t]{6}{*}{ East Africa } & & 33 & $29(87.88)$ & $2(6.06)$ & $2(6.06)$ \\
\hline & Ethiopia & 3 & $1(33.33)$ & $1(33.33)$ & $1(33.33)$ \\
\hline & Somalia & 1 & $0(0)$ & $1(100.00)$ & $0(0)$ \\
\hline & Rwanda & 1 & $1(100.00)$ & $0(0)$ & $0(0)$ \\
\hline & Tanzania & 17 & $17(100.00)$ & $0(0)$ & $0(0)$ \\
\hline & Uganda & 11 & $10(90.91)$ & $0(0)$ & $1(9.09)$ \\
\hline \multirow[t]{2}{*}{ North Africa } & & 4 & $3(75.00)$ & $1(25.00)$ & $0(0)$ \\
\hline & Sudan & 4 & $3(75.00)$ & $1(25.00)$ & $0(0)$ \\
\hline \multirow[t]{6}{*}{ South Africa } & & 54 & $48(88.89)$ & $5(9.26)$ & $1(1.85)$ \\
\hline & Mozambique & 11 & $11(100.00)$ & $0(0)$ & $0(0)$ \\
\hline & Angola & 30 & $24(80.00)$ & $5(16.67)$ & $1(3.33)$ \\
\hline & Malawi & 5 & $5(100.00)$ & $0(0)$ & $0(0)$ \\
\hline & Republic of south Africa & 4 & $4(100.00)$ & $0(0)$ & $0(0)$ \\
\hline & Zambia & 4 & $4(100.00)$ & $0(0)$ & $0(0)$ \\
\hline \multirow[t]{15}{*}{ West Africa } & & 260 & $177(68.08)$ & $70(26.92)$ & $13(5.00)$ \\
\hline & Republic of Niger & 10 & $8(80.00)$ & $2(20.00)$ & $0(0)$ \\
\hline & Ghana & 27 & $27(100.00)$ & $0(0)$ & $0(0)$ \\
\hline & Benin & 8 & $4(50.00)$ & $2(25.00)$ & $2(25.00)$ \\
\hline & Burkina Faso & 2 & $2(100.00)$ & $0(0)$ & $0(0)$ \\
\hline & Côte d'Ivoire & 21 & $13(61.90)$ & $6(28.57)$ & $2(9.52)$ \\
\hline & Guinea & 48 & $39(81.25)$ & $9(18.75)$ & $0(0)$ \\
\hline & Guinea-Bissau & 2 & $1(50.00)$ & $1(50.00)$ & $0(0)$ \\
\hline & Liberia & 5 & $1(20.00)$ & $3(60.00)$ & $1(20.00)$ \\
\hline & Mali & 2 & $2(100.00)$ & $0(0)$ & $0(0)$ \\
\hline & Mauritania & 3 & $2(66.67)$ & $0(0)$ & $1(33.33)$ \\
\hline & Nigeria & 118 & $74(62.71)$ & $37(31.36)$ & $7(5.93)$ \\
\hline & Sierra Leone & 11 & $3(27.27)$ & $8(72.73)$ & $0(0)$ \\
\hline & Senegal & 1 & $0(0)$ & $1(100.00)$ & $0(0)$ \\
\hline & Togo & 2 & $1(50.00)$ & $1(50.00)$ & $0(0)$ \\
\hline \multirow[t]{3}{*}{ Southeast Asia } & & 2 & $1(50.00)$ & $1(50.00)$ & $0(0)$ \\
\hline & Philippines & 1 & $0(0)$ & $1(100.00)$ & $0(0)$ \\
\hline & Myanmar & 1 & $1(100.0)$ & $0(0)$ & $0(0)$ \\
\hline \multirow[t]{2}{*}{ Oceania } & & 4 & $1(25.00)$ & $3(75.00)$ & $0(0)$ \\
\hline & Papua New Guinea & 4 & $1(25.00)$ & $3^{a}(75.00)$ & $0(0)$ \\
\hline Total & & 471 & $342(72.61)$ & 107 (22.72) & $22(4.67)$ \\
\hline
\end{tabular}

a The 3 samples harboured SVMNT haplotypes 


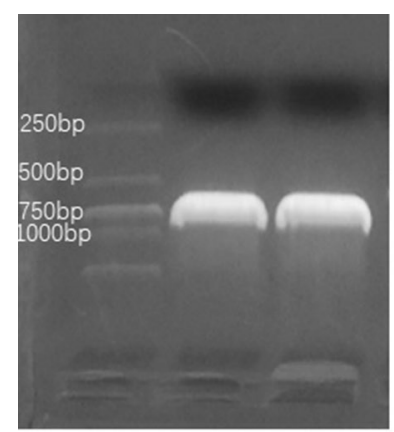

$k 13$

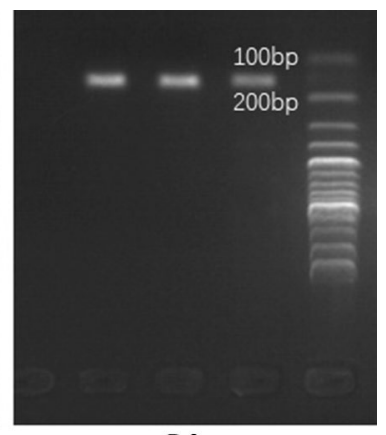

Pfort

Fig. 2 Electropherograms of $k 13$ and Pfcrt genotypes detected in imported P. falciparum cases countries in Africa represented almost $50 \%$ of all malaria cases worldwide: Nigeria (25\%), The Democratic Republic of Congo (11\%), Mozambique (5\%), and Uganda (4\%) [1]. Furthermore, African countries spread the infection to other countries, including France $(>2500)$, China (>2000), UK (approximately 1500), and Germany (>500) in 2017 [1, 20]. Zhejiang province, located in southeast China, had several cases of $P$. falciparum malaria imported from Africa in recent years [21, 22]. The current study evaluated drug resistance markers of $P$. falciparum infections predominantly from Africa using molecular assays to advance drug policies against malaria.

Chloroquine, an easy-to-use and affordable first-line antimalarial agent, is comprehensively used globally to

Table 2 Mutant types observed in the $k 13$ of imported $P$. falciparum infections

\begin{tabular}{|c|c|c|c|c|c|}
\hline Codon position & $\begin{array}{l}\text { Amino acid } \\
\text { reference }\end{array}$ & $\begin{array}{l}\text { Nucleotide } \\
\text { reference }\end{array}$ & $\begin{array}{l}\text { Amino acid } \\
\text { mutation }\end{array}$ & $\begin{array}{l}\text { Nucleotide } \\
\text { mutation }\end{array}$ & Location and year (No.) \\
\hline 441 & $P$ & ACC & $S$ & AIC & Nigeria $2017(1)^{a}, 2018(1)^{b}$ \\
\hline 464 & $\mathrm{D}$ & GAT & $E$ & GAEA & Guinea 2018 (1) \\
\hline 469 & C & TGC & C & TGI & Nigeria 2017 (1) \\
\hline 471 & $\mathrm{R}$ & CGT & $\mathrm{R}$ & CG $\underline{C}$ & Angola $2016(1)$ \\
\hline 478 & $\mathrm{~T}$ & $A C C$ & $\mathrm{~T}$ & $A C \underline{G}$ & Benin 2017 (1) \\
\hline 492 & $L$ & TTA & $L$ & TTG & Ghana 2016 (1) \\
\hline 493 & Y & TAC & Y & TAI & Liberia 2017 (1), Côte d'Ivoire 2018 (1) \\
\hline 496 & G & GGT & G & GGE & Ghana 2018 (1) \\
\hline 503 & K & AAG & $E$ & $\underline{G} A G$ & Cameroon 2018 (1) \\
\hline 535 & $\mathrm{~T}$ & $A C G$ & $\mathrm{~T}$ & $A C \underline{A}$ & Côte d'Ivoire 2017 (1) \\
\hline 561 & $\mathrm{R}$ & CGT & $\mathrm{H}$ & CAT & Rwanda 2017 (1), Myanmar 2017 (1) \\
\hline 578 & A & $\mathrm{GCT}$ & $S$ & ICT & Cameroon $2016(1)$ \\
\hline 589 & V & GTC & V & GTG & Nigeria 2016 (2) \\
\hline 622 & $\mathrm{R}$ & AGA & 1 & AIA & Mozambique 2016 (1), Somalia 2016 (1) \\
\hline 638 & G & GGA & G & GGG & Republic of Niger 2017 (1) \\
\hline 650 & V & GTT & $\mathrm{F}$ & ITT & Nigeria $2016(1)$ \\
\hline 664 & N & AAT & $\mathrm{N}$ & $\mathrm{AAC}$ & Nigeria 2017 (1) \\
\hline 676 & A & GCC & A & $\mathrm{GCI}$ & Nigeria 2016 (1), Guinea 2016 (1) \\
\hline 694 & $\mathrm{~N}$ & AAT & K & $\mathrm{AAG}$ & Côte d'Ivoire 2017 (1), Angola 2017 (1) \\
\hline
\end{tabular}

No. number of isolates

a Isolate with mutant type of Pfcrt

b Isolate with mixed type of Pfcrt

Table 3 Genetic diversity of $\boldsymbol{k} 13$ gene from imported $P$. falciparum infections

\begin{tabular}{|c|c|c|c|c|c|c|c|c|}
\hline Region & $n$ & P-distance & $\mathrm{S}$ & $\mathrm{Hd}(\mathrm{SD})$ & $\mathrm{Pi}(J C)$ & k & Tajima's D & $p$-value ${ }^{\Delta}$ \\
\hline West Africa & 247 & 0.000 & 15 & $0.141(0.030)$ & 0.0002 & 0.162 & -2.32586 & $<0.01$ \\
\hline South Africa & 53 & 0.000 & 3 & $0.111(0.059)$ & 0.00014 & 0.113 & -1.68986 & $>0.05$ \\
\hline Central Africa & 106 & 0.000 & 2 & $0.038(0.026)$ & 0.00005 & 0.038 & -1.37037 & $>0.10$ \\
\hline East Africa & 21 & 0.000 & 2 & $0.186(0.110)$ & 0.00024 & 0.190 & -1.51414 & $>0.10$ \\
\hline Total $^{\mathrm{a}}$ & 427 & 0.000 & 20 & $0.114(0.021)$ & 0.00016 & 0.126 & -2.38774 & $<0.01$ \\
\hline
\end{tabular}

S number of segregating sites, $\mathrm{Hd}$ haplotype diversity, $\mathrm{Pi}(J C)$ nucleotide diversity (Jukes and Cantor), $k$ average number of nucleotide differences

a Sequences from North Africa, Southeast Asia and Oceania were not included due to their limited sample size or number of mutants

$\triangle p$-value for Tajima's D 
treat $P$. falciparum and Plasmodium vivax. Nevertheless, this drug was withdrawn from most endemic countries due to the high levels of resistance, which has resulted in a two- or threefold increase in malaria deaths and hospital admission for severe malaria in various African countries $[19,23]$. Over ten multi-mutations sharing the common K76T substitution have been detected and are associated with CQR in field and laboratory strains of P. falciparum [4, 24, 25]. Five major haplotypes at Pfcrt residues 72-76 (CVIET, SVMNT, SVIET, CVMNT, and CVTNT) were related to CQR, and CVIET and SVMNT were regarded as the most resistant haplotypes [7, 2628]. CVIET is predominant in Africa, whereas SVMNT is more common in South America [27]. Our study confirmed that CVIET was the most common mutation type in infections imported from Africa, similar to previous studies $[26,28]$. The high frequency of SVMNT in Papua New Guinea was also consistent with other studies [29]. It was demonstrated that the spatial distribution of mutant alleles was mainly related to local drug policy. The increase in the prevalence of SVMNT isolates in Tanzania from $0 \%(0 / 156)$ in 2003 to $3.68 \%(6 / 163)$ in 2004 was due to selective pressure for amodiaquine resistance [27]. Therefore, it was postulated that CVIET might be displaced by SVMNT in African regions where amodiaquine was increasingly used [27, 30]. However, our study refuted this hypothesis by showing the absence of SVMNT double mutants in Tanzania and other African countries. The disagreement in the results may be due to differences in sample size and survey sites. The present results indicate that parasites with CVIET are still a major threat in Africa.

With the cessation of CQ use between 1998 and 2008, parasite populations with wild-type CVMNK returned progressively, demonstrated by the increased frequency of this haplotype in Africa [30-33]. For instance, the frequency of a CQS genotype increased from $28.0 \%$ in 2003 to $53.7 \%$ in 2012 after disuse of CQ for 9 years in Cameroon [34]. This hypothesis is supported in our study by the consistent detection of CVMNK (72.61\%) in imported malaria cases and by another study wherein most $P$. falciparum isolates from Africa harbored wildtype alleles [33]. A possible explanation is that CVIET mutants were not fit enough to evolve into wide-type strains without selective pressure [30]. These results help understand the dynamics of major Pfcrt haplotypes in Africa and enrich the evidence for drug policy.

K13 propeller polymorphisms in $P$. falciparum have been widely studied since it was first described in GMS [12]. Molecular markers for $k 13$ have been identified and can help elucidate artemisinin delayed parasite clearance [15]. In this study, 19 alleles were identified, and eight (P441S, D464E, K503E, R561H, A578S, R622I, V650F, and
N694K) were nonsynonymous. Similarly with previous literatures, the propeller domain of the $k 13$ gene showed a limited diversity of alleles in Africa $[18,35,36]$. Of note is that one of the validated $k 13$ resistance mutations$\mathrm{R} 561 \mathrm{H}$ - was detected in two patients, one each from Rwanda and Myanmar. R561H in Myanmar and western Thailand, although not the predominantly popular, was more prevalent than that in Africa [33, 37, 38]. The only infected patient from Myanmar was positive for R561H. Previous studies found the $\mathrm{R} 561 \mathrm{H}$ allele in Congo. However, $\mathrm{R} 561 \mathrm{H}$ might be the first time found from Rwanda $[36,37,39]$. In addition, mutation A578S identified in one isolate from Cameroon, was also found in other African countries, including Gabon, Uganda, Mali, Kenya and DR Congo [18, 39-41], but it was not associated with clinical or in vitro resistance to artemisinin according to previous studies $[37,42]$. The study also found the uncommon $k 13$ mutation R622I in two cases, one in Mozambique and one in Somalia, and this mutation was initially reported in northwest Ethiopia at the border with Sudan [43]. A previous study showed that, of a total of over 14,000 screened samples from 59 countries in Africa, Asia, Oceania, and South America, only one sample from Zambia was positive for R622I [37]. Interestingly, one out of three patients from Ethiopia bearing R622I mutation showed day-3 positivity in Giemsa-stained smears [43]. Further clinical studies are required to investigate the role of R622I in acquired resistance to artemisinin.

In Nigeria, where nearly $25 \%$ of the samples collected in this study originated, seven samples exhibited six mutant types (two nonsynonymous types comprising P441S and $\mathrm{V} 650 \mathrm{~F}$, and four nonsynonymous types comprising C469C, V589V, N664N, and A676A). Similarly, no validated or candidate amino acid mutations were detected in southwestern Nigeria according to recent studies $[35,44]$. However, a low prevalence of single nucleotide polymorphisms (G665C, V666V, P553P, V510V, A578S, $\mathrm{D} 464 \mathrm{~N}$, and $\mathrm{Q} 613 \mathrm{H})$ were also reported, although they differed from the results obtained in the current study $[35,44]$. In general, these results indicate that the profiles of the molecular markers conferring artemisinin delayed clearance were still optimistic with respect to the public health situation in terms of malaria in Nigeria.

The molecular genetic analyses conducted in this study showed that the haplotype diversity of the samples imported Africa was relatively low. Similarly, haplotype diversity of P. falciparum isolates in Congo, Ghana, Kenya and Tanzania in a previous study was $0.067,0.123$, 0.066 and 0.056 , respectively [45]. Also, southwestern Nigeria reported Hd $0.080-0.157$ in two states [35]. However, another research reported Hd 0.74 from African isolates [46]. The difference might result from heterogeneity of spatial distribution of samples. The current study 
also showed that the sequence diversity varied among the isolates from West, South, East, and Central Africa. The isolates from East Africa had the highest genetic diversity, whereas those from Central Africa had the lowest diversity. The discrepancy might have been related to the unequal sample sizes in different areas. Further investigation is required. Regarding the neutrality test, there was no consistent results. Tajima's D value in this study was found to be negative and statistically significantly in terms of the total samples from Africa. Another research also reported similar trends from African isolates [46]. Nevertheless, negative Tajima's D value without statistical significance was observed in Nigeria which indicated $k 13$ gene evolved under neutral model [35].

\section{Conclusions}

In conclusion, this study determined the frequency and spatial distribution of $P f c r t$ and $k 13$ mutations associated with drug resistance in $P$. falciparum malaria cases imported into Zhejiang province. In Africa, wild-type Pfcrt was predominant, but detection of $k 13$ mutants was limited by the absence of genetically validated molecular markers of artemisinin delayed parasite clearance, except one case from Rwanda harbouring the $\mathrm{R} 561 \mathrm{H}$ mutation. Both Pfcrt and k13 mutations were detected in patients from Southeast Asia and Oceania. These results may guide efforts to make more rational and targeted drug policies to eliminate resistant malaria in the study region and demonstrated that molecular markers are an effective and convenient tool to improve the surveillance of drug resistance.

\section{Supplementary information}

Supplementary information accompanies this paper at https://doi. org/10.1186/s12936-020-3140-0.

Additional file 1: Table S1. Primers for Pfcrt and K13 propeller genotyping assay. Table S2. Distribution of imported P. falciparum cases of Zhejiang province between 2016 and 2018.

\section{Abbreviations}

Pfcrt: Plasmodium falciparum chloroquine resistance; CQ: Chloroquine; CQR: Chloroquine resistance; CQS: Chloroquine sensitive; k13: Kelch13; ACT: Artemisinin-based combination therapy; GMS: Greater Mekong subregion.

\section{Acknowledgements}

We are grateful to the physicians and staff of the Prefecture and Local Center for Disease Control and Prevention for their invaluable assistance in sample collection and epidemiological investigation.

\section{Authors' contributions}

XXW, QYL, XYF and HY carried out the molecular studies. SSZ and FH conceived the study. XXW and WR analysed the data and drafted the manuscript. All authors read and approved the final manuscript.

\section{Funding}

This work was funded by the National Important Scientific \& Technological Project (2018ZX10101002-002), National Health Commission Special Project for Lancang-Mekong Cooperation in 2017 (No. 2020399), Natural Science Foundation of Shanghai (No. 18ZR1443400), and National Institutes of Health/National Institute of Allergy and Infectious Diseases U19 project (No. U19Al129386)

\section{Availability of data and materials}

The datasets analysed in this study are available from the corresponding author on reasonable request.

\section{Ethics approval and consent to participate}

This study was approved by the Ethical Review Committee of Zhejiang Provincial Center for Disease Control and Prevention.

\section{Consent for publication}

Not applicable.

\section{Competing interests}

The authors declare that they have no competing interests.

\section{Author details}

${ }^{1}$ National Institute of Parasitic Diseases, Chinese Center for Disease Control and Prevention, Key Laboratory of Parasite and Vector Biology, Schistosomiasis and Filariasis, $\mathrm{MOH}$, and WHO Collaborating Centre for Malaria, Shanghai, People's Republic of China. ${ }^{2}$ Zhejiang Provincial Center for Disease Control and Prevention, Zhejiang, People's Republic of China.

Received: 30 August 2019 Accepted: 24 January 2020

Published online: 04 February 2020

\section{References}

1. WHO. world malaria report. Geneva, World Health Organization; 2018.

2. Harinasuta T, Suntharasamai P, Viravan C. Chloroquine-resistant falciparum malaria in Thailand. Lancet. 1965;2:657-60.

3. Andrea E, Lehane AM, Jérôme C, Fidock DA. PfCRT and its role in antimalarial drug resistance. Trends Parasitol. 2012;28:504-14.

4. Viswanathan L, Bray PG, Dominik VP, Johnson DJ, Paul H, Muhle RA, et al. A critical role for PfCRT K76T in Plasmodium falciparum verapamil-reversible chloroquine resistance. Embo J. 2005;24:2294-305.

5. Picot S, Olliaro P, Monbrison FD, Bienvenu AL, Price RN, Ringwald P. A systematic review and meta-analysis of evidence for correlation between molecular markers of parasite resistance and treatment outcome in falciparum malaria. Malar J. 2009;8:89.

6. Awasthi G, Prasad GB, Das A. Population genetic analyses of Plasmodium falciparum chloroquine receptor transporter gene haplotypes reveal the evolutionary history of chloroquine-resistant malaria in India. Int J Parasitol. 2011:41:705-9.

7. Awasthi G, Prasad GB, Das A. Pfcrt haplotypes and the evolutionary history of chloroquine-resistant Plasmodium falciparum. Mem Inst Oswaldo Cruz. 2012;107:129-34.

8. Osman ME, Mockenhaupt FP, Bienzle U, Elbashir MI, Giha HA. Field-based evidence for linkage of mutations associated with chloroquine (pfcrt/ pfmdr1) and sulfadoxine-pyrimethamine (pfdhfr/pfdhps) resistance and for the fitness cost of multiple mutations in P. falciparum. Infect Genet Evol. 2007:7:52-9.

9. Sidhu AB, Verdier-Pinard D, Fidock DA. Chloroquine resistance in Plasmodium falciparum malaria parasites conferred by pfcrt mutations. Science. 2002:298:210-3.

10. Sisowath C, Petersen I, Veiga MI, Mårtensson A, Premji Z, Bjorkman A, et al. In vivo selection of Plasmodium falciparum parasites carrying the chloroquine-susceptible pfcrt K76 allele after treatment with artemetherlumefantrine in Africa. J Infect Dis. 2009;199:750-7.

11. WHO. Guidelines for the treatment of malaria. Geneva, World Health Organization; 2006

12. Harald N, Youry S, Kurt S, Smith BL, Duong S, Fukuda MM. Evidence of artemisinin-resistant malaria in western Cambodia. N Engl J Med. 2008;359:2619-20. 
13. Dondorp AM, Nosten F, Yi P, Das D, Phyo AP, Tarning J, et al. Artemisinin resistance in Plasmodium falciparum malaria. N Engl J Med. 2009:361:455-67.

14. WHO. Artemisinin resistance and artemisinin-based combination therapy efficacy. Geneva, World Health Organization; 2018.

15. WHO. Artemisinin and artemisinin-based combination therapy resistance. Geneva, World Health Organization; 2017.

16. Lu F, Culleton R, Zhang M, Ramaprasad A, von Seidlein L, Zhou H, et al. Emergence of indigenous artemisinin-resistant Plasmodium falciparum in Africa. N Engl J Med. 2017;376:991-3.

17. Zhang T, Xu X, Jiang J, Yu C, Tian C, Li W. Surveillance of antimalarial resistance molecular markers in imported Plasmodium falciparum malaria cases in Anhui, China, 2012-2016. Am J Trop Med Hyg. 2018;98:1132-6.

18. Voumbo-Matoumona DF, Kouna LC, Madamet M, Maghendji-Nzondo S, Pradines B, Lekana-Douki JB. Prevalence of Plasmodium falciparum antimalarial drug resistance genes in Southeastern Gabon from 2011 to 2014. Infect Drug Resist. 2018;11:1329-38.

19. Trape JF. The public health impact of chloroquine resistance in Africa. Am J Trop Med Hyg. 2001;64:12-7.

20. Feng J, Zhang L, Huang F, Yin JH, Tu H, Xia ZG, et al. Ready for malaria elimination: zero indigenous case reported in the People's Republic of China. Malar J. 2018;17:315.

21. Zhang X, Yao L, Sun J, Pan J, Chen H, Zhang L, et al. Malaria in Southeastern China from 2012 to 2016: analysis of imported cases. Am J Trop Med Hyg. 2018;98:1107-12

22. Chen H, Yao L, Zhang L, Zhang X, Lu Q, Yu K, et al. Malaria in Zhejiang Province, China, from 2005 to 2014. Am J Trop Med Hyg. 2015;93:305-9.

23. Ocan M, Akena D, Nsobya S, Kamya MR, Senono R, Kinengyere AA, et al. Persistence of chloroquine resistance alleles in malaria endemic countries: a systematic review of burden and risk factors. Malar J. 2019;18:76.

24. Warhurst DC. Polymorphism in the Plasmodium falciparum chloroquine-resistance transporter protein links verapamil enhancement of chloroquine sensitivity with the clinical efficacy of amodiaquine. Malar J. 2003;2:31.

25. Holmgren G, Gil JP, Ferreira PM, Veiga MI, Obonyo CO, Bjorkman A. Amodiaquine resistant Plasmodium falciparum malaria in vivo is associated with selection of pfcrt 76T and pfmdr1 86Y. Infect Genet Evol. 2006;6:309-14.

26. Gadalla NB, Tavera G, Mu J, Kabyemela ER, Fried M, Duffy PE, et al. Prevalence of Plasmodium falciparum anti-malarial resistance-associated polymorphisms in pfcrt, pfmdr1 and pfnhe1 in Muheza, Tanzania, prior to introduction of artemisinin combination therapy. Malar J. 2015;14:129.

27. Alifrangis M, Dalgaard MB, Lusingu JP, Vestergaard LS, Staalsoe T, Jensen AT, et al. Occurrence of the Southeast Asian/South American SVMNT haplotype of the chloroquine-resistance transporter gene in Plasmodium falciparum in Tanzania. J Infect Dis. 2006;193:1738-41.

28. Bray PG, Martin RE, Tilley L, Ward SA, Kirk K, Fidock DA. Defining the role of PfCRT in Plasmodium falciparum chloroquine resistance. Mol Microbiol. 2005;56:323-33.

29. Barnadas C, Timinao L, Javati S, Iga J, Malau E, Koepfli C, et al. Significant geographical differences in prevalence of mutations associated with Plasmodium falciparum and Plasmodium vivax drug resistance in two regions from Papua New Guinea. Malar J. 2015;14:399.

30. Sa JM, Twu O, Hayton K, Reyes S, Fay MP, Ringwald P, et al. Geographic patterns of Plasmodium falciparum drug resistance distinguished by differential responses to amodiaquine and chloroquine. Proc Natl Acad Sci USA. 2009;106:18883-9.

31. Wang X, Mu J, Li G, Chen P, Guo X, Fu L, et al. Decreased prevalence of the Plasmodium falciparum chloroquine resistance transporter 76T marker associated with cessation of chloroquine use against $P$. falciparum malaria in Hainan, People's Republic of China. Am J Trop Med Hyg. 2005;72:410-4.
32. Golassa L, Kamugisha E, Ishengoma DS, Baraka V, Shayo A, Baliraine FN, et al. Identification of large variation in pfcrt, pfmdr-1 and pfubp-1 markers in Plasmodium falciparum isolates from Ethiopia and Tanzania. Malar J. 2015;14:264.

33. Zhou RM, Zhang HW, Yang CY, Liu Y, Zhao YL, Li SH, et al. Molecular mutation profile of pfcrt in Plasmodium falciparum isolates imported from Africa in Henan province. Malar J. 2016;15:265.

34. Ndam NT, Basco LK, Ngane VF, Ayouba A, Ngolle EM, Deloron P, et al. Reemergence of chloroquine-sensitive pfcrt K76 Plasmodium falciparum genotype in southeastern Cameroon. Malar J. 2017;16:130.

35. Oboh MA, Ndiaye D, Antony HA, Badiane AS, Singh US, Ali NA, et al. Status of artemisinin resistance in malaria parasite Plasmodium falciparum from molecular analyses of the Kelch13 gene in Southwestern Nigeria. BioMed Res Int. 2018;2018:2305062.

36. Tacoli C, Gai PP, Bayingana C, Sifft K, Geus D, Ndoli J, et al. Artemisinin resistance-associated K13 polymorphisms of Plasmodium falciparum in Southern Rwanda, 2010-2015. Am J Trop Med Hyg. 2016;95:1090-3.

37. Menard D, Khim N, Beghain J, Adegnika AA, Shafiul-Alam M, Amodu O, et al. A worldwide map of Plasmodium falciparum K13-propeller polymorphisms. N Engl J Med. 2016;374:2453-64.

38. Bonnington CA, Phyo AP. Plasmodium falciparum Kelch 13 mutations and treatment response in patients in Hpa-Pun District, Northern Kayin State, Myanmar. Malar J. 2017;16:480.

39. Taylor SM, Parobek CM, DeConti DK, Kayentao K, Coulibaly SO, Greenwood BM, et al. Absence of putative artemisinin resistance mutations among Plasmodium falciparum in Sub-Saharan Africa: a molecular epidemiologic study. J Infect Dis. 2015;211:680-8.

40. Ouattara A, Kone A, Adams M, Fofana B, Maiga AW, Hampton S, et al. Polymorphisms in the K13-propeller gene in artemisinin-susceptible Plasmodium falciparum parasites from Bougoula-Hameau and Bandiagara, Mali. Am J Trop Med Hyg. 2015;92:1202.

41. Muwanguzi J, Henriques G, Sawa P, Bousema T, Sutherland CJ, Beshir KB. Lack of $k 13$ mutations in Plasmodium falciparum persisting after artemisinin combination therapy treatment of Kenyan children. Malar J. 2016;15:36

42. Kamau E, Campino S, Amenga-Etego L, Drury E, Ishengoma D, Johnson $\mathrm{K}$, et al. K13-propeller polymorphisms in Plasmodium falciparum parasites from sub-Saharan Africa. J Infect Dis. 2015;11:1352-5.

43. Bayih AG, Getnet G, Alemu A, Getie S, Mohon AN, Pillai DR. A unique Plasmodium falciparum K13 gene mutation in Northwest Ethiopia. Am J Trop Med Hyg. 2016;94:132-5.

44. Igbasi U, Oyibo W, Omilabu S, Quan H, Chen SB, Shen HM, et al. Kelch 13 propeller gene polymorphism among Plasmodium falciparum isolates in Lagos, Nigeria: molecular epidemiologic study. Trop Med Int Health. 2019;24:1011-7.

45. Mita T, Culleton R, Takahashi N, Nakamura M, Tsukahara T, Hunja CW, et al. Little polymorphism at the k13 propeller locus in worldwide Plasmodium falciparum populations prior to the introduction of artemisinin combination therapies. Antimicrob Agents Chemother. 2016;60:3340-7.

46. Pacheco MA, Kadakia ER, Chaudhary Z, Perkins DJ, Kelley J, Ravishankar $\mathrm{S}$, et al. Evolution and genetic diversity of the $\mathrm{k} 13$ gene associated with artemisinin delayed parasite clearance in Plasmodium falciparum. Antimicrob Agents Chemother. 2019;63:e02550-18.

\section{Publisher's Note}

Springer Nature remains neutral with regard to jurisdictional claims in published maps and institutional affiliations. 